\title{
Acute sleep deprivation and the selective consolidation of emotional memories
}

\author{
Ivan Vargas, ${ }^{1,2}$ Jessica D. Payne, ${ }^{3}$ Alexandria Muench, ${ }^{2,4}$ Kate R. Kuhlman, ${ }^{5,6}$ \\ and Nestor L. Lopez-Duran ${ }^{7}$
}

${ }^{1}$ Center for Sleep and Circadian Neurobiology, University of Pennsylvania, Philadelphia, Pennsylvania 19104, USA; ${ }^{2}$ Behavioral Sleep Medicine Program, Department of Psychiatry, University of Pennsylvania, Philadelphia, Pennsylvania 19104, USA; ${ }^{3}$ Department of Psychology, University of Notre Dame, Notre Dame, Indiana 46556, USA; ${ }^{4}$ Department of Psychology, Philadelphia College of Osteopathic Medicine, Philadelphia, Pennsylvania 19131, USA; ${ }^{5}$ Department of Psychology and Social Behavior, University of California Irvine, Irvine, California 92612, USA; ${ }^{6}$ Cousins Center for Psychoneuroimmunology, University of California Los Angeles, Los Angeles, California 90095, USA; 7 Department of Psychology, University of Michigan, Ann Arbor, Michigan 48109, USA

\begin{abstract}
Research suggests that sleep preferentially consolidates the negative aspects of memories at the expense of the neutral aspects. However, the mechanisms by which sleep facilitates this emotional memory trade-off remain unknown. Although active processes associated with sleep-dependent memory consolidation have been proposed to underlie this effect, this trade-off may also be modulated by non-sleep-related processes, such as the circadian factors, stress-related factors, and/or mood congruent context effects involved in sleep deprivation. We sought to examine the potential role of these factors by randomizing 39 young adults into either a total sleep deprivation condition (26 consecutive hours awake) or a sleep condition ( $8 \mathrm{~h}$ sleep opportunity). Replicating the emotional memory trade-off effect, negative objects were better remembered than neutral objects or background images. However, in spite of generally worse memory performance (for neutral and background information), sleep-deprived participants showed similar recognition rates for negative emotional memories relative to participants who were given a full night of sleep.
\end{abstract}

Sleep plays a vital role in preferentially consolidating the emotional aspects of memories at the expense of neutral background information (Payne and Kensinger 2010, 2018; Bennion et al. 2015a). This emotional memory trade-off is assessed using a scenes task, where participants study scenes consisting of either a negative (e.g., snake) or neutral (e.g., chipmunk) object on a neutral background (e.g., a forest; Payne et al. 2008a). While this trade-off has been observed following even very brief delays (i.e., $30 \mathrm{~min}$; Payne et al. 2008a), it is magnified by an intervening period of sleep. Specifically, individuals are more likely to remember the negative aspects of scenes at the cost of forgetting the neutral aspects following a night of sleep, or even a daytime nap, compared to an equal period of daytime wakefulness (Payne et al. 2008a, 2015). Because these findings have been linked to active sleepbased processes (e.g., nocturnal REM sleep and delta activity during the nap), these studies suggest that sleep "actively" and "selectively" promotes the consolidation of information that is emotionally salient and therefore likely to be adaptive (Payne and Kensinger 2010). Recent work suggests that tagging mechanisms operating at encoding to identify salient information (Payne and Kensinger 2018), interact with the unique, high-frequency rhythms of sleep (e.g., theta activity during REM sleep, and the hippocampal sharp wave-ripples and sleep spindles that are embedded in slow oscillations) to selectively reactivate (Boyce et al. 2017; Feld and Born 2017) and perhaps downscale (Tononi and Cirelli 2014) memory representations, which may lead to preferential remembering.

Although previous studies have begun to identify potential mechanisms underlying this selective memory benefit, it remains unclear whether the selective emotional memory consolidation that occurs during the nocturnal period is specifically related to

Corresponding author: ivargas@pennmedicine.upenn.edu Article is online at http://www.learnmem.org/cgi/doi/10.1101/lm.049312.119. processes that are unique to sleep. To date, few studies have investigated whether the emotional memory benefit can be observed after a similar time period (i.e., overnight), but in the absence of sleep. If such a benefit can be observed in the absence of sleep, this would suggest that it can be modulated by other, non-sleeprelated, processes (e.g., circadian factors, stress-related factors, and/or mood-congruent context effects). Some studies suggest this might be the case. For example, Sterpenich et al. (2007) reported data suggesting memory for negative, but not positive or neutral, stimuli was still intact following a night of total sleep deprivation and comparable to memory performance following a night of sleep. Alternatively, if the emotional memory benefit is not observed in the absence of sleep, it would further support the idea that sleep-related processes specifically modulate this effect. For example, Wagner et al. (2001) demonstrated that emotional memories were preferentially consolidated following a 3-h period of late night, REM-rich sleep, as compared to an equal period of late night wakefulness, and Payne et al. (2012) showed that the emotional memory benefit in the trade-off task correlated positively with time spent in REM sleep. According to these findings, the nocturnal consolidation of emotional memories is sleepspecific (and possibly REM-sleep specific). In the present study, we build on these findings by comparing the relative impact of a full night of sleep and a full night of wakefulness on the magnification of the emotional memory trade-off effect (i.e., the selective consolidation of negative objects). A between groups experimental

\footnotetext{
(C) 2019 Vargas et al. This article is distributed exclusively by Cold Spring Harbor Laboratory Press for the first 12 months after the full-issue publication date (see http://learnmem.cshlp.org/site/misc/terms.xhtml). After 12 months, it is available under a Creative Commons License (AttributionNonCommercial 4.0 International), as described at http://creativecommons. org/licenses/by-nc/4.0/.
} 
design was used to evaluate the extent to which there was a difference between conditions (sleep versus deprivation) in the selective consolidation of emotional memories. We hypothesized that there would be a greater emotional memory trade-off in the sleep condition, as compared to the sleep deprivation condition (i.e., the memory trade-off is sleep-specific).

Participants included 39 young adults (20 females; $\mathrm{M}_{\mathrm{age}}=$ $22.6, \mathrm{SD}_{\text {age }}=3.1$ ). While the majority of participants in the final sample identified as Caucasian (56.4\%), the overall composition of the sample was racially diverse. Additional details regarding the participants and other aspects of this study can be reviewed elsewhere (e.g., exclusion criteria, withdrawal rates, exact racial distribution, and other outcome variables; Vargas and Lopez-Duran 2017). In brief, each participant was invited to complete an in-laboratory baseline evaluation (see Fig. 1 for Study Protocol). Specifically, they completed a series of online questionnaires about their sleep habits, mood, and stress. The self-report measures assessed: demographic and lifestyle information (e.g., habitual caffeine use, alcohol use), general sleep patterns (Pittsburgh Sleep Quality Index; Buysse et al. 1989), daytime sleepiness (Epworth Sleepiness Scale; Johns 1991), chronobiological preference (Morningness-Eveningness Questionnaire; Horne and Ostberg 1975), insomnia symptoms (Insomnia Severity Index; Morin 1993), depressive symptoms (Patient Health Questionnaire; Kroenke et al. 2001), anxiety and worry (Generalized Anxiety Disorder Screener; Spitzer et al. 2006), and perceived stress (Perceived Stress Scale; Cohen et al. 1983). At the conclusion of the baseline visit, participants were given an actigraphy device (Actiwatch 2, Philips Respironics) and instructed to wear it during the subsequent week as a means of assessing habitual sleep patterns.

Participants returned to the laboratory $\sim 1$ wk after the baseline laboratory visit (Fig. 1). All participants stayed in the laboratory during two consecutive weekend nights. Beginning at 12:00 A.M., participants were instructed to be in bed with the lights off (lights out). The first night (adaptation night) served to familiarize participants with sleeping in a novel environment. They were asked to get out of bed immediately after awakening (lights on); however, any participants still sleeping at 8:00 a.m. were awoken by the research staff. The encoding portion of the memory task was completed on the second night (experimental night). Participants were randomly assigned to either the total sleep deprivation (TSD; $n=20)$ or sleep condition $(n=19)$. Participants were blind to their group assignment prior to the experimental night. During the experimental night, participants assigned to the sleep condition were given another 8-h sleep opportunity. Participants assigned to the TSD condition underwent a night of sleep deprivation (approximately 26 consecutive hours awake). The recognition portion of the memory task was completed the next morning (10:00 a.m.). Participants also completed self-report measures of sleepiness (Stanford Sleepiness Scale; Hoddes et al. 1973) and positive and negative affect that morning (Positive and Negative Affect Scale; Watson et al. 1988).

The memory trade-off task (Kensinger et al. 2006, 2007) consisted of either a negative (e.g., a crashed car) or neutral object (e.g., an intact car) on a plausible neutral background scene (e.g., a

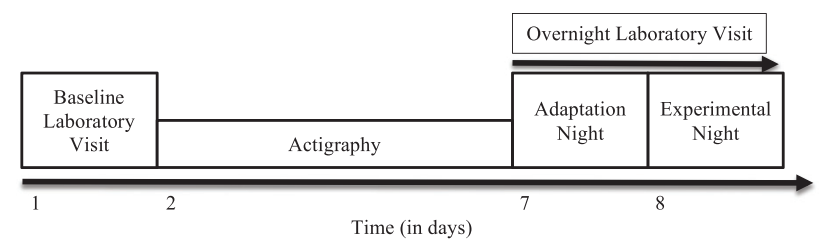

Figure 1. Study protocol. street). For each of 64 scenes (e.g., a car on a street), eight different versions were created by placing each of two similar neutral objects (e.g., two images of a car) and each of two related negative objects (e.g., two images of a crashed car) on each of two neutral backgrounds (e.g., two images of a street). An additional 32 scenes served as "new" items on a recognition memory test. The scenes task was administered using E-Prime 2.0 software (Psychology Software Tools) on an IBM-compatible computer and the images were presented on a 17 -inch Dell computer monitor. During the encoding session (between 10:00-11:00 p.m. on the experimental night), participants viewed a set of 64 scenes ( 32 with a neutral object and 32 with a negative object, all on neutral backgrounds) for 5 sec each, and then indicated on a seven-point scale whether they would approach or move away from the scene if they encountered it in real life. The studied version of each scene (of the eight possible versions) was counterbalanced across participants. In the selfpaced recognition task, which occurred at 10:00 a.m. the next morning, participants viewed objects and backgrounds, presented separately and one at a time. This memory test used a combination of items that were either identical (i.e., the exact same objects and backgrounds seen at encoding) or, alternatively, similar but nonidentical (e.g., a picture of a snake highly similar to the snake seen at encoding) to studied items. Participants either saw the identical or similar version of a particular item at test, never both, and were asked to indicate whether each item was the same as the one seen previously, similar (but not identical) to the one seen previously, or entirely new. Items included in the recognition test were: 32 identical objects, 32 similar objects, and 32 new objects (16 negative and 16 neutral, each), as well as 32 identical backgrounds, 32 similar backgrounds (16 previously shown with a negative item and 16 with a neutral item, each), and 32 new backgrounds.

As in the original studies by Payne and Kensinger (e.g., Kensinger et al. 2007; Payne et al. 2008a), an overall recognition score for each valence and scene type was calculated by taking the proportion of both "same" and "similar" responses to "same" items. As a preliminary step, the association between each relevant covariate (e.g., perceived stress, habitual sleep patterns) and mean recognition scores was assessed in order to determine which covariates should be included in the final analyses. We next conducted a 2 (condition: sleep, TSD) $\times 2$ (valence: negative, neutral) $\times 2$ (scene component: object, background) mixed analysis of variance (SPSS MIXED) on mean recognition scores. Condition, valence and scene component were entered as fixed effects, whereas only valence and scene component were entered into the model as repeated effects. The MIXED procedure is commonly used when analyzing mixed-effects and/or repeated measures ANOVA and can replicate a GLM procedure when no random effect is included. We used the MIXED procedure instead of GLM because it allows for the analysis of incomplete data (subject-wise deletion) as well as for the specification of the covariance structure (Littell et al. 1998). In the present study, mean recognition scores were organized by each combination of valence and scene type (i.e., 2 valence types $\times 2$ scene types $=4$ mean recognition scores), and because within-subject recognition scores are likely correlated, the models can be more precise if within-subject covariance is accounted for. In addition to this, Bayesian statistics were computed using the summary statistics module in JASP as an alternative way to quantify the degree to which that the data is a better fit to the alternative hypothesis as compared to the null hypothesis (Ly et al. 2018). To stay consistent with the original study by Payne et al. (2008a), because the false alarm rate "same" or "similar" responses to new items) was low ( $<5 \%$ for "same" responses to new items and $<20 \%$ for "similar" responses to new items), and importantly, did not differ between conditions (all $P s>0.60$ ), we used uncorrected recognition scores in our primary analyses. The 
Table 1. Group differences for all preexperiment variables

\begin{tabular}{|c|c|c|c|c|}
\hline & Sleep $(n=19)$ & $\operatorname{TSD}(n=20)$ & $F_{(\mathrm{df})}$ & $P$-value \\
\hline Age & $22.9(3.3)$ & $22.3(3.0)$ & $0.35_{(1,37)}$ & 0.56 \\
\hline Anxiety (GAD-7) & $2.1(1.9)$ & $1.9(1.9)$ & $0.16_{(1,37)}$ & 0.74 \\
\hline Depression (PHQ-9) & $1.6(1.7)$ & $2.2(1.6)$ & $1.17_{(1,37)}$ & 0.29 \\
\hline Perceived Stress (PSS) & $9.2(4.7)$ & $13.3(5.7)$ & 5.96 & 0.02 \\
\hline Insomnia (ISI) & $4.2(3.6)$ & $5.1(3.8)$ & $0.50_{(1,37)}$ & 0.46 \\
\hline Sleepiness (ESS) & $6.3(3.5)$ & $6.2(4.8)$ & $0.002_{(1,37)}$ & 0.96 \\
\hline Sleep quality (PSQI) & $4.6(2.6)$ & $4.6(2.3)$ & $0.001_{(1,37)}$ & 0.98 \\
\hline Chronotype (MEQ) & $44.4(6.3)$ & $47.3(8.0)$ & $1.54(1,37)$ & 0.22 \\
\hline Caffeine use, $n(\%)$ & & & $0.29_{(1,37)}$ & 0.59 \\
\hline 0 drinks & $4(21.1)$ & $5(25.0)$ & & \\
\hline $1-2$ drinks & $13(68.4)$ & $14(70.0)$ & & \\
\hline 3-4 drinks & $2(10.5)$ & $1(5.0)$ & & \\
\hline Alcohol use, n (\%) & & & $1.70_{(1,37)}$ & 0.20 \\
\hline Never & $4(21.1)$ & $2(10.0)$ & & \\
\hline Once per month or less & $7(36.8)$ & $6(30.0)$ & & \\
\hline Once per week & $6(31.6)$ & $6(30.0)$ & & \\
\hline More than once per week & $1(5.3)$ & $6(30.0)$ & & \\
\hline Every day & $1(5.3)$ & $0(0)$ & & \\
\hline \multicolumn{5}{|l|}{ Sleep continuity, mean (SD) } \\
\hline TST, $\min$ & $420.2(40.2)$ & $388.4(40.9)$ & 5.48 & 0.03 \\
\hline $\mathrm{SE}, \%$ & $90.9(2.9)$ & $90.6(3.5)$ & $0.09(1,34)$ & 0.77 \\
\hline $\mathrm{SL}, \min$ & $14.4(17.9)$ & $8.5(6.0)$ & $1.83(1,34)$ & 0.19 \\
\hline WASO, $\min$ & $41.8(13.7)$ & $39.8(15.2)$ & $0.17(1,34)$ & 0.68 \\
\hline NWAK, \# & $23.4(7.8)$ & $22.0(7.8)$ & $0.29_{(1,34)}$ & 0.59 \\
\hline
\end{tabular}

GAD-7, Generalized Anxiety Disorder Screener; PHQ-9, Patient Health Questionnaire; PSS, Perceived Stress Scale; ISI, Insomnia Severity Index; ESS, Epworth Sleepiness Scale; PSQI, Pittsburgh Sleep Quality Index; MEQ, Morningness Eveningness Questionnaire; TST, Total Sleep Time; SE, Sleep Efficiency; SL, Sleep Latency; WASO, Wake After Sleep Onset; NWAK, Nocturnal Awakenings. Actigraphy (i.e., sleep continuity) data was missing for three participants.

unadjusted effects, however, were also computed using the corrected recognition scores and are reported below. Finally, trade-off scores were calculated by subtracting the memory score for the associated backgrounds from the memory score of the objects (e.g., proportion recognized for negative objects minus proportion recognized for backgrounds on which negative items were presented). These trade-off scores assess the magnitude of the difference between object and background memory (Payne et al. 2015).

Although randomization procedures were used, participants in the TSD condition reported greater perceived stress (PSS) and lower average total sleep time (TST; assessed via actigraphy) during the baseline week. There were no group differences on any other measure (see Table 1 for all estimates). Follow-up analyses were conducted to evaluate the unadjusted effect (i.e., condition and the interaction with condition were not entered as fixed effects) of PSS and TST on memory. There were no statistically significant associations between PSS or TST and mean recognition scores and thus were not included in the subsequent models, PSS, $F_{(1,149)}=0.052$, $P=0.82$, PSS $\times$ scene component, $F_{(1,149)}=0.50, P=0.48, \mathrm{PSS} \times \mathrm{va}-$ lence, $F_{(1,149)}=0.34, P=0.56$, three-way interaction, $F_{(1,149)}=0.08$, $P=0.78$, TST, $F_{(1,123)}=2.43, P=0.12$, TST $\times$ scene component, $F_{(1,123)}=0.95, P=0.33$, TST $\times$ valence, $F_{(1,123)}=3.74, P=0.06$, threeway interaction, $F_{(1,123)}=0.002, P=0.97$. Relative to participants in the sleep condition, participants in the TSD condition also reported greater subjective sleepiness, greater negative affect, and lower positive affect in the morning (see Table 2). As with PSS and TST, unadjusted analyses were conducted to assess the association between sleepiness/affect and recognition scores. Greater self-reported sleepiness was associated with a lower overall recognition score, $\left.F_{(1,142)}=17.36, P<0.001\right)$, whereas greater positive affect was associated with a higher overall recognition score, $F_{(1,142)}=10.36, P<$ 0.01 , irrespective of valence or scene component. All other effects were nonsignificant.

The final unadjusted model included condition, scene component, and valence (and their interactions) as fixed effects, whereas the adjusted models included sleepiness and positive affect as covariates (see Table 3). According to the unadjusted model, there was a significant effect of condition and scene component but not

Table 2. Group differences in sleepiness, affect, and sleep continuity

\begin{tabular}{|c|c|c|c|c|}
\hline & \multicolumn{4}{|c|}{ Condition } \\
\hline & Sleep $(n=19)$ & $\operatorname{TSD}(n=20)$ & $F_{(\mathrm{df})}$ & $P$-value \\
\hline Sleepiness (SSS), mean (SD) & $3.3(0.9)$ & $5.4(0.9)$ & $54.72_{(1,35)}$ & $<0.001$ \\
\hline \multicolumn{5}{|l|}{ Affect (PANAS), mean (SD) } \\
\hline Negative affect & $10.5(1.0)$ & $12.6(2.6)$ & $11.28(1.35)$ & $<0.01$ \\
\hline Positive affect & $15.4(5.6)$ & $12.2(3.2)$ & $4.31_{(1,35)}$ & 0.05 \\
\hline \multicolumn{5}{|l|}{ Sleep continuity, mean (SD) } \\
\hline TST, $\min$ & $444.9(24.0)$ & - & - & - \\
\hline $\mathrm{SE}, \%$ & $91.6(5.7)$ & _- & - & _- \\
\hline $\mathrm{SL}, \min$ & $12.0(17.7)$ & - & - & - \\
\hline WASO, $\min$ & $8.1(20.8)$ & - & - & - \\
\hline NWAK, \# & $4.5(11.5)$ & - & - & - \\
\hline
\end{tabular}

Sleepiness and affect were assessed in the morning following the experimental night. Sleep continuity was assessed via actigraphy during the experimental night. SSS, Stanford Sleepiness Scale; PANAS, Positive and Negative Affect Scale. Actigraphy (i.e., sleep continuity) data was missing for three participants. 


\begin{tabular}{|c|c|c|c|}
\hline & $F$ & $P$-value & $\mathrm{BF}_{10}^{\mathrm{a}}$ \\
\hline \multicolumn{4}{|l|}{ Unadjusted model } \\
\hline Condition & 9.18 & $<0.01$ & 1.28 \\
\hline Scene component & 22.27 & $<0.001$ & 965.40 \\
\hline Valence & 3.06 & 0.08 & 0.79 \\
\hline Condition $\times$ Scene component & 0.07 & 0.79 & 0.38 \\
\hline Condition $\times$ Valence & 0.02 & 0.89 & 0.34 \\
\hline Scene component $\times$ Valence & 17.89 & $<0.001$ & 25.56 \\
\hline Condition $\times$ Scene component $\times$ Valence & 0.59 & 0.44 & 0.39 \\
\hline \multicolumn{4}{|l|}{ Adjusted model } \\
\hline Sleepiness & 4.20 & 0.04 & 1.50 \\
\hline Positive affect & 3.03 & 0.08 & 1.04 \\
\hline Condition & 0.001 & 0.98 & 0.32 \\
\hline Scene component & 20.72 & $<0.001$ & 464.65 \\
\hline Valence & 3.30 & 0.07 & 0.61 \\
\hline Condition $\times$ Scene component & 0.04 & 0.85 & 0.36 \\
\hline Condition $\times$ Valence & 0.06 & 0.81 & 0.32 \\
\hline Scene component $\times$ Valence & 16.32 & $<.001$ & 15.76 \\
\hline Condition $\times$ Scene component $\times$ Valence & 0.43 & 0.52 & 0.37 \\
\hline
\end{tabular}

a Bayes factors were also computed using the summary statistics module in JASP; greater values indicating more evidence for the alternative hypothesis and lower values indicating more evidence for the null hypothesis (Ly et al. 2018).

valence on overall recognition. ${ }^{8}$ That is, participants in the TSD condition, compared to those in the sleep condition, had lower overall recognition rates, irrespective of valence or scene component, and participants, across both conditions, were more likely to remember objects relative to background images (see Table 3 for all model estimates). There was also a significant two-way interaction between valence and scene component (Fig. 2). Specifically, for objects, there was a difference in recognition rates by valence, such that negative objects were better remembered compared to neutral objects. The three-way interaction was not statistically significant, indicating that the emotional memory trade-off was not specific to sleep. Moreover, the magnitude of the difference between object and background memory (i.e., trade-off effect) was also assessed in both groups. The magnitude of the trade-off effect in the TSD condition, $M=0.27$, was not significantly different from the magnitude of the effect in the sleep condition, $M=0.21, F_{(1,73)}$ $=1.07, P=0.31$, suggesting that a period of nocturnal wakefulness, and/or factors associated with acute sleep deprivation, produces the same selective memory benefit for negative components of scenes as a night of sleep.

While, in general, the results remained mostly unchanged when the main effects of self-reported positive affect and sleepiness were entered into the model, the main effect of condition on overall memory was no longer statistically significant (Table 3). In addition, correcting for false alarms did not meaningfully change the results. Corrected recognition scores were computed by subtracting the combined false alarm rate (proportion of "same" and "similar" responses to "new" stimuli) from the uncorrected overall recogni-

\footnotetext{
${ }^{8}$ As a matter of protocol, the memory variable was also separated into specific and general recognition memory for each scene component. A response was scored as specific recognition of visual details when a participant correctly responded "same" to a "same" item, and as general recognition without specific details when a participant responded "similar" to a "same" item. The results were similar to overall recognition. For this reason and because this publication is a brief communication, these data were omitted and are available upon request.
}

tion score (proportion of both "same" and "similar" responses to "same" items). The unadjusted model with corrected recognition scores entered as the dependent variable also showed a main effect of condition, $F_{(1,154)}=4.88, P=0.03$ and a significant valence by scene component interaction across conditions, $F_{(1,154)}=6.43, P=$ 0.01 . All other effects in this model were nonsignificant, scene component, $F_{(1,154)}=0.001, P=>0.20$, valence, $F_{(1,154)}=0.07, P=>0.20$, scene component $\times$ condition, $F_{(1,154)}=0.0003, P=>0.20$, valence $\times$ condition, $F_{(1,154)}=0.06, P=>0.20$, three-way interaction, $F_{(1,154)}=$ $0.11, P=>0.20$.

Our findings suggest that negative objects, relative to neutral objects and backgrounds, are preferentially consolidated into memory following an intervening period of nocturnal sleep or nocturnal wakefulness/sleep deprivation. This is consistent with past studies showing that humans generally show preferential consolidation of negative aspects of scenes compared to the neutral, background aspects (Kensinger et al. 2007). The emotional memory trade-off, however, was not greater in magnitude in the sleep condition compared to the deprivation condition. This is in contrast to the many studies that show preferential emotional memory consolidation during nocturnal sleep compared to daytime wakefulness (e.g., $\mathrm{Hu}$ et al. 2006; Payne et al. 2008a). Furthermore, despite the overall decrease in recognition rates for the TSD condition, the recognition rates for negative objects was relatively unaffected, which highlights the robustness of memory for emotional aspects of scenes. This finding is in line with previous research showing that memory for negative stimuli is particularly resilient to the otherwise deleterious effect of sleep deprivation on memory (Atienza and Cantero 2008; Tempesta et al. 2015).

While the precise explanation for these findings is uncertain, there are at least three potential explanations regarding why the emotional memory trade-off was observed in both conditions: (1) mood congruent context effects, (2) circadian effects, and (3) sleep deprivation induced stress/cortisol effects. With respect to mood-congruent context effects, it is possible that participants in the TSD condition more accurately recognized stimuli that were consistent with their affective state, in this case, the negative objects (Blaney 1986). As shown here and in previous studies (Pilcher et al. 1996; Scott et al. 2006), sleep deprivation is associated with greater levels of negative affect and a greater tendency to rate images as more negative (Tempesta et al. 2015). With respect to circadian effects, Payne et al. (2015) previously showed that the emotional memory trade-off was also observed following an afternoon nap relative to closely matched periods of daytime wakefulness, which helps rule out circadian factors as a major contributor to the effect. It is possible, however, that the circadian effects on emotional memory extend beyond the memory processes that are utilized in the short-term (i.e., 30 min daytime nap), and only affect

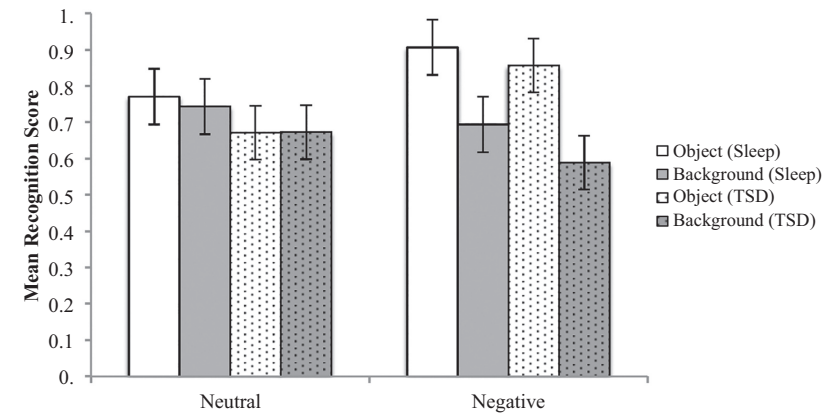

Figure 2. Mean overall recognition scores by valence and scene type for both conditions. Overall recognition is the proportion of both "same" and "similar" responses to "same" items. Error bars represent the 95\% confidence interval. 
those utilized in long-term consolidation (i.e., overnight). Additionally, there may be something about the "middle of the night" that enhances long-term memory consolidation (e.g., increases in cortisol at night), regardless of whether one is sleeping during that time (Van Marle et al. 2013; Bennion et al. 2015b). With respect to sleep-deprivation-related stress, it is possible the selective consolidation of emotional stimuli can also be modulated by acute stress/cortisol release. Prior research evaluating the effect of sleep on emotional memory consolidation has primarily utilized daytime wakefulness as the comparison group (e.g., Payne et al. 2008a, 2012). An important distinction between a daytime wakefulness condition and a nighttime wakefulness condition is that nighttime wakefulness (as operationalized in the present study) included acute sleep deprivation, which likely elicits a cognitive and/ or physiological stress response (Leproult et al. 1997; Vargas and Lopez-Duran 2017). A stress response can also enhance memory encoding and consolidation (Nadel et al. 2002; Lupien et al. 2007), particularly of negative stimuli (Payne et al. 2007; Cunningham et al. 2018), and therefore, a sleep-deprivation-induced stress response may potentially explain why the selective consolidation of emotional memories was observed following TSD.

These findings should be considered in the context of a number of study limitations. For example, it is important to consider that acute sleep deprivation (i.e., one night of complete nocturnal wakefulness) has limited ecological validity, and is associated with a number of post-deprivation effects that are not directly observed following an equivalent period of daytime wakefulness (e.g., changes in stress reactivity, autonomic functioning, and mood; Meerlo et al. 2008). These post-deprivation effects may markedly contribute to emotional memories being preferentially consolidated (as opposed to naturally occurring circadian effects), and thus, should be the focus of future research. Moreover, this study represents data from a relatively small sample, predominantly composed of highly educated young adults, and therefore, limits generalizability. While not necessarily a limitation, it is important to highlight that our definition of overall recognition included "similar" responses to stimuli that were previously seen. We chose to report information from this most lenient memory measure as opposed to specific recognition (correct responses were only those in which the participant responded "same" to a "same" item). A central component of the emotional memory trade-off is that emotionally valenced stimuli enhance memory for the general aspects of the stimuli, also known as "gist" memory (Kensinger et al. 2007; Payne et al. 2008b). This has been shown to be especially true when the stimulus is a complex scene (Kensinger et al. 2006). In order to assess the impact of sleep or sleep deprivation on the emotional memory trade-off, it is important to consider and/or allow for the possibility that only general aspects of the objects are encoded/consolidated into memory.

In summary, while our study replicated the emotional memory trade-off observed in previous experiments (Payne et al. 2008a; Payne and Kensinger 2010, 2018; Bennion et al. 2015a), it was not specific to participants in the sleep condition. Similar recognition rates for negative objects were observed among acutely sleepdeprived participants, suggesting that the selective consolidation of emotional memories may not necessarily be sleep dependent, and that there may be multiple pathways by which emotional memories are preferentially consolidated at night.

\section{References}

Atienza M, Cantero JL. 2008. Modulatory effects of emotion and sleep on recollection and familiarity. J Sleep Res 17: 285-294. doi:10.1111/j .1365-2869.2008.00661.x

Bennion KA, Payne JD, Kensinger EA. 2015a. Selective effects of sleep on emotional memory: what mechanisms are responsible? Transl Issues Psychol Sci 1: 79-88. doi:10.1037/tps0000019
Bennion KA, Steinmetz KRM, Kensinger EA, Payne JD. 2015b. Sleep and cortisol interact to support memory consolidation. Cereb Cortex 25: 646657. doi:10.1093/cercor/bht255

Blaney PH. 1986. Affect and memory: a review. Psychol Bull 99: 229. doi:10 $.1037 / 0033-2909.99 .2 .229$

Boyce R, Williams S, Adamantidis A. 2017. REM sleep and memory. Curr Opin Neurobiol 44: 167-177. doi:10.1016/j.conb.2017.05.001

Buysse DJ, Reynolds CF, Monk TH, Berman SR, Kupfer DJ. 1989. The Pittsburgh Sleep Quality Index: a new instrument for psychiatric practice and research. Psychiatry Res 28: 193-213. doi:10.1016/ 0165-1781(89)90047-4

Cohen S, Kamarck T, Mermelstein R. 1983. A global measure of perceived stress. J Health Soc Behav 24: 385-396. doi:10.2307/2136404

Cunningham TJ, Leal SL, Yassa MA, Payne JD. 2018. Post-encoding stress enhances mnemonic discrimination of negative stimuli. Learn Mem 25: 611-619. doi:10.1101/lm.047498.118

Feld GB, Born J. 2017. Sculpting memory during sleep: concurrent consolidation and forgetting. Curr Opin Neurobiol 44: 20-27. doi:10 .1016/j.conb.2017.02.012

Hoddes E, Zarcone V, Smythe H, Phillips R, Dement WC. 1973. Quantification of sleepiness: a new approach. Psychophysiology 10: 431436. doi:10.1111/j.1469-8986.1973.tb00801.x

Horne JA, Ostberg O. 1975. A self-assessment questionnaire to determine morningness-eveningness in human circadian rhythms. Int J Chronobiol 4: 97-110.

Hu P, Stylos-Allan M, Walker MP. 2006. Sleep facilitates consolidation of emotional declarative memory. Psychol Sci 17: 891-898.

Johns MW. 1991. A new method for measuring daytime sleepiness: the Epworth sleepiness scale. Sleep 14: 540-545. doi:10.1093/sleep/14.6.540

Kensinger EA, Garoff-Eaton RJ, Schacter DL. 2006. Memory for specific visual details can be enhanced by negative arousing content. J Mem Lang 54: $99-112$. doi:10.1016/j.jml.2005.05.005

Kensinger EA, Garoff-Eaton RJ, Schacter DL. 2007. Effects of emotion on memory specificity: memory trade-offs elicited by negative visually arousing stimuli. J Mem Lang 56: 575-591. doi:10.1016/j.jml.2006.05 arousin

Kroenke K, Spitzer RL, Williams JBWW. 2001. The PHQ-9: validity of a brief depression severity measure. J Gen Intern Med 16: 605-613. doi:10.1046/j 1525-1497.2001.016009606.x

Leproult R, Copinschi G, Buxton O, Van Cauter E. 1997. Sleep loss results in an elevation of cortisol levels the next evening. Sleep 20: 865-870. doi:10.1093/sleep/20.10.865

Littell RC, Henry PR, Ammerman CB. 1998. Statistical analysis of repeated measures data using SAS procedures. J Anim Sci 76: 1216-1231. doi:10 $.2527 / 1998.7641216 \mathrm{x}$

Lupien SJ, Maheu F, Tu M, Fiocco A, Schramek TE. 2007. The effects of stress and stress hormones on human cognition: implications for the field of brain and cognition. Brain Cogn 65: 209-237. doi:10.1016/j.bandc.2007 .02 .007

Ly A, Raj A, Etz A, Marsman M, Gronau QF, Wagenmakers E-J. 2018. Bayesian reanalyses from summary statistics: a guide for academic consumers. Adv Methods Pract Psychol Sci 1: 367-374. doi:10.1177/ 2515245918779348

Meerlo P, Sgoifo A, Suchecki D. 2008. Restricted and disrupted sleep: effects on autonomic function, neuroendocrine stress systems and stress responsivity. Sleep Med Rev 12: 197-210. doi:10.1016/j.smrv.2007.07 respon

Morin CM. 1993. Insomnia: psychological assessment and management. Guilford Press, New York.

Nadel L, Payne JD, Jacobs WJ. 2002. The relationship between episodic memory and context: clues from memory errors made while under stress. Physiol Res 51: 3-11.

Payne JD, Kensinger EA. 2010. Sleep's role in the consolidation of emotional episodic memories. Curr Dir Psychol Sci 19: 290-295. doi:10.1177/ 0963721410383978

Payne JD, Kensinger EA. 2018. Stress, sleep, and the selective consolidation of emotional memories. Curr Opin Behav Sci 19: 36-43. doi:10.1016/j .cobeha.2017.09.006

Payne JD, Jackson ED, Hoscheidt S, Ryan L, Jacobs WJ, Nadel L. 2007. Stress administered prior to encoding impairs neutral but enhances emotional long-term episodic memories. Learn Mem 14: 861-868. doi:10.1101/lm .743507

Payne J, Ellenbogen J, Walker M, Stickgold R. 2008b. The role of sleep in memory consolidation. In Learning and memory: a comprehensive reference and concise learning and memory: the editor's selection (ed. JH Byrne), pp. 547-569. Elsevier, Oxford, UK.

Payne JD, Stickgold R, Swanberg K, Kensinger EA. 2008a. Sleep preferentially enhances memory for emotional components of scenes. Psychol Sci 19: 781-788. doi:10.1111/j.1467-9280.2008.02157.x

Payne JD, Chambers AM, Kensinger EA. 2012. Sleep promotes lasting changes in selective memory for emotional scenes. Front Integr Neurosci 6: 108 . doi:10.3389/fnint.2012.00108 
Payne JD, Kensinger EA, Wamsley EJ, Spreng RN, Alger SE, Gibler K, Schacter DL, Stickgold R. 2015. Napping and the selective consolidation of negative aspects of scenes. Emotion 15: 176-186. doi:10.1037/ a0038683

Pilcher JJ, Horne JA, Huffcutt A. 1996. Effects of sleep deprivation on performance: a meta-analysis. Sleep 19: 318-326. doi:10.1093/sleep/19 .4 .318

Scott JPR, McNaughton LR, Polman RCJ. 2006. Effects of sleep deprivation and exercise on cognitive, motor performance and mood. Physiol Behav 87: 396-408. doi:10.1016/j.physbeh.2005.11.009

Spitzer RL, Kroenke K, Williams JBW, Löwe B. 2006. A brief measure for assessing generalized anxiety disorder: the GAD-7. Arch Intern Med 166: 1092-1097. doi:10.1001/archinte.166.10.1092

Sterpenich V, Albouy GG, Boly MM, Vandewalle G, Darsaud A, Balteau E, Dang-Vu TT, Desseilles M, D'Argembeau A, Gais S, et al. 2007.

Sleep-related hippocampo-cortical interplay during emotional memory recollection. PLoS Biol 5: 2709-2722. doi:10.1371/journal.pbio .0050282

Tempesta D, De Gennaro L, Natale V, Ferrara M. 2015. Emotional memory processing is influenced by sleep quality. Sleep Med 16: 862-870. doi:10 .1016/j.sleep.2015.01.024
Tononi G, Cirelli C. 2014. Sleep and the price of plasticity: from synaptic and cellular homeostasis to memory consolidation and integration. Neuron 81: 12-34. doi:10.1016/j.neuron.2013.12.025

Van Marle HJF, Hermans EJ, Qin S, Overeem S, Fernández G. 2013. The effect of exogenous cortisol during sleep on the behavioral and neural correlates of emotional memory consolidation in humans.

Psychoneuroendocrinology 38: 1639-1649. doi:10.1016/j.psyneuen.2013 .01 .009

Vargas I, Lopez-Duran N. 2017. Investigating the effect of acute sleep deprivation on hypothalamic-pituitary-adrenal-axis response to a psychosocial stressor. Psychoneuroendocrinology 79: 1-8. doi:10.1016/j .psyneuen.2017.01.030

Wagner U, Gais S, Born J. 2001. Emotional memory formation is enhanced across sleep intervals with high amounts of rapid eye movement sleep. Learn Mem 8: 112-119.

Watson D, Clark L, Tellegen A. 1988. Development and validation of brief measures of positive and negative affect: the PANAS scales. J Pers Soc Psychol 54: 1063-1070. doi:10.1037/0022-3514.54.6.1063

Received January 8, 2019; accepted in revised form April 16, 2019. 


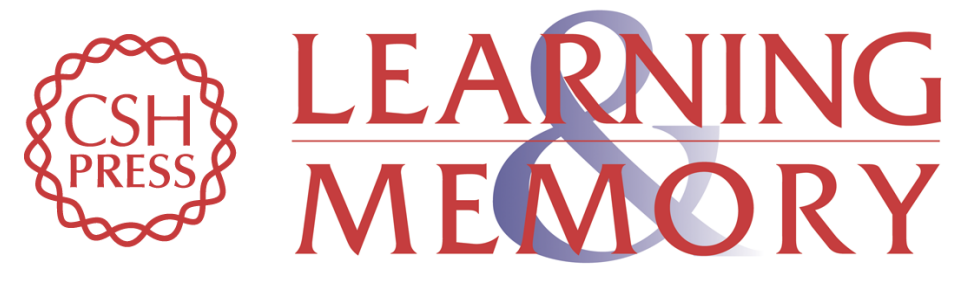

\section{Acute sleep deprivation and the selective consolidation of emotional memories}

Ivan Vargas, Jessica D. Payne, Alexandria Muench, et al.

Learn. Mem. 2019, 26:

Access the most recent version at doi:10.1101/Im.049312.119

References This article cites 38 articles, 3 of which can be accessed free at:

http://learnmem.cshlp.org/content/26/6/176.full.html\#ref-list-1

Creative This article is distributed exclusively by Cold Spring Harbor Laboratory Press for the

Commons

first 12 months after the full-issue publication date (see

License http://learnmem.cshlp.org/site/misc/terms.xhtml). After 12 months, it is available under a Creative Commons License (Attribution-NonCommercial 4.0 International), as described at http://creativecommons.org/licenses/by-nc/4.0/.

Email Alerting Receive free email alerts when new articles cite this article - sign up in the box at the Service top right corner of the article or click here. 